\title{
Tipificación de dos nombres del género Verbascum incluidos en la subsect. Singuliflora (Scrophulariaceae)
}

\author{
P. P. FERRER-GALLEGO ${ }^{1}$, R. ROSELLÓ ${ }^{2}$, E. LAGUNA $^{1} \&$ J. B. PERIS ${ }^{2}$ \\ ${ }^{1}$ Servicio de Vida Silvestre, Centro para la Investigación y Experimentación Forestal de la Generalitat Valenciana \\ (CIEF), av. Comarques del País Valencià, 114, ES-46930 Quart de Poblet, España \\ ${ }^{2}$ Departament de Botànica, Facultad de Farmàcia, Universitat de València, av. Vicent Andrés Estellés, s/n, ES-46100 \\ Burjassot, España
}

ORCID iD. P. P. FERRER-GALLEGO: http://orcid.org/0000-0001-7595-9302, R. ROSELLÓ: http://orcid.org/0000-0002-1146-8149, E. LAGUNA: http://orcid.org/0000-0002-9674-2767, J. B. PERIS: http://orcid.org/0000-0001-9580-2660

Autor para correspondencia: P. P. Ferrer-Gallego (flora.cief@gva.es)

Editor: J. M. Montserrat

Recibido 15 enero 2019; aceptado 22 noviembre 2019; publicado on line 6 abril 2020

\begin{abstract}
Typification of two names of the genus Verbascum included in Subsect. Singuliflora (Scrophulariaceae).- The nomenclatural types of Verbascum barnadesii and V. fontqueri (三Celsia valentina) (Scrophulariaceae) are designated
\end{abstract} from specimens preserved at $\mathrm{C}$ and $\mathrm{BC}$, respectively.

Key words: Celsia; nomenclature; Scrophulariaceae; typification.

\section{Resumen}

TipificACIÓN DE DOS NOMBRES DEL GÉNERo VERBASCUM INCLUIDOS EN LA SUBSECT. SINGULIFLORA (SCROPHULARIACEAE).- Se designan los tipos nomenclaturales de Verbascum barnadesii y V. fontqueri (三 Celsia valentina) (Scrophulariaceae) a partir de especímenes conservados en los herbarios $\mathrm{C}$ y BC, respectivamente.

Palabras clave: Celsia; nomenclatura; Scrophulariaceae; tipificación.

\section{Cómo citar este artículo / Citation}

Ferrer-Gallego, P. P., Roselló, R., Laguna, E. \& Peris, J. B. 2020. Tipificación de dos nombres del género Verbascum incluidos en la subsect. Singuliflora Murb. (Scrophulariaceae). Collectanea Botanica 39: e004. https://doi.org/10.3989/ collectbot.2020.v39.004

\section{Copyright}

(C) 2020 CSIC. This is an open-access article distributed under the terms of the Creative Commons Attribution 4.0 International (CC BY 4.0) License. 


\section{INTRODUCCIÓN}

El género Verbascum L. pertenece a la tribu Verbasceae (Scrophulariaceae) y comprende entre 300 y 325 especies distribuidas por el hemisferio boreal, excepto en Norteamérica. Su mayor centro de especiación y diversificación se encuentra en las regiones balcánica e iranoturania (Murbeck, 1933; Ferguson, 1972; Heywood, 1993; Judd et al., 2002; Benedí, 2009).

Como resultado de la consulta y estudio del material original de dos nombres de este género pertenecientes a la subsect. Singuliflora Murb. de la sect. Verbascum (= género Celsia L.), V. barnadesii Vahl y $V$. fontqueri Benedí \& J. M. Monts. (三 Celsia valentina Font Quer), y la bibliografía donde se ha tratado en profundidad estas dos especies, tanto desde el punto de vista taxonómico como nomenclatural (Murbeck, 1921, 1925, 1933, 1939; Benedí \& Montserrat, 1985; Benedí et al., 1989; Benedí, 2009), llegamos a la conclusión que ambos nombres están todavía por tipificar.

Así, el objetivo del presente trabajo es fijar la aplicación de estos nombres mediante su tipificación a partir del estudio del material original conservado en diferentes herbarios. Respecto al nombre $V$. fontqueri, Benedí \& Montserrat (1985) realizaron una propuesta de tipificación, pero desafortunadamente no puede ser aceptada por ser contraria a lo dispuesto en el Código Internacional de Nomenclatura (Turland et al., 2018), según se especifica más adelante.

Verbascum barnadesii es una especie endémica de la Península Ibérica, presente en su centro y cuadrante suroccidental, donde crece en claros de matorral, bordes de caminos, taludes y carreteras, sobre suelos arenosos preferentemente ácidos (Benedí, 2009). Por su parte, $V$. fontqueri es un endemismo de área restingida a la provincia de Valencia, con óptimo de distribución en las sierras litorales y sublitorales, pero con poblaciones en territorios del interior del centro y sur de la provincia, donde crece en herbazales y matorrales aclarados, pastos secos y márgenes de caminos (Aguilella et al., 2010; Benedí, 2009). Resulta una especie incluida dentro del Anexo II "especies protegidas no catalogadas" del listado valenciano de especies protegidas de flora (Generalitat Valenciana, 2013: Orden 6/2013, de 25 de marzo, de la Conselleria de Infraestructuras, Territorio y Medio Ambiente, disponible en: http://www.dogv.gva.es/portal/ficha disposicion_pc.jsp?sig=003163/2013\&L=1).

\section{RESULTADOS Y DISCUSIÓN}

\section{Verbascum barnadesii}

El protólogo de $V$. barnadesii Vahl (1791: 39) incluye una concisa frase-diagnosis "VERBASCUM caule subnudo, foliis lanceolatis dentato-sinuatis glabris, pedunculis unifloris", junto a una descripción más amplia de la planta, y una diagnosis para su diferenciación frente a dos especies "Verbascum Osbekii differt caule folioso, foliis caulinis oblongis petiolatis dentate-incisis, pedunculis calycibusque lanatis. Verbascum sinuatum distinguitur foliis radicalibus obtusissimis tomentosis, floribus sessilibus aggregatis". Además, el protólogo también incluye la siguiente información: "Habitat in colliculis de Hincapie versus oppidum Ortaleza tractus Madritensis. Specimen mecum communicavit Cel. Dn. Barnades filius inventoris".

En el herbario del Royal Botanic Gardens de Kew (K) se conservan dos especímenes relevantes para la tipificación de este nombre, con códigos de barras K000806392 y K000806393. El espécimen K000806392 es un fragmento de planta compuesto por una inflorescencia con flores y dos hojas de la parte basal de la planta (Fig. 1). Este espécimen se encuentra en mal de estado de conservación, y junto al material vegetal se conserva una etiqueta de revisión de Murbeck, fechada en 1937, en la que aparece la palabra "cotypus". El espécimen K000806393, está montado en el mismo pliego de herbario que el espécimen anterior, pero en una hoja diferente, y se compone de dos fragmentos bastante

\footnotetext{
La definición del término "cotypus" fue publicada por Thomas (1893). Bajo el concepto de cotipo se englobaba a uno de los dos o más ejemplares que juntos forman la base de una especie, no habiendo sido elegido ninguno como tipo. En este sentido, ningún nombre tendría a la vez tipo y cotipo, o bien un único tipo o dos o más cotipos. Esta categoría no es considerada en la actualidad en el ICN (Turland et al., 2018), ni tampoco aparece definido el término, ya que éste quedaría incluido bien dentro de la categoría de síntipo, si el material ha sido citado en el protólogo, o bien dentro del concepto de material original del autor, es decir, conjunto de ejemplares e ilustraciones entre los cuales se puede designar un lectótipo. Por otra parte, otros autores consideran el término cotipo [incluido el de clastótipo en el sentido de Swingle (1912)] como un fragmento o espécimen duplicado del tipo, es decir, isótipo (ICN Art. 9.5), isolectótipo, isoneótipo o isoepítipo (ICN nota al pie del Art. 9.4).
} 


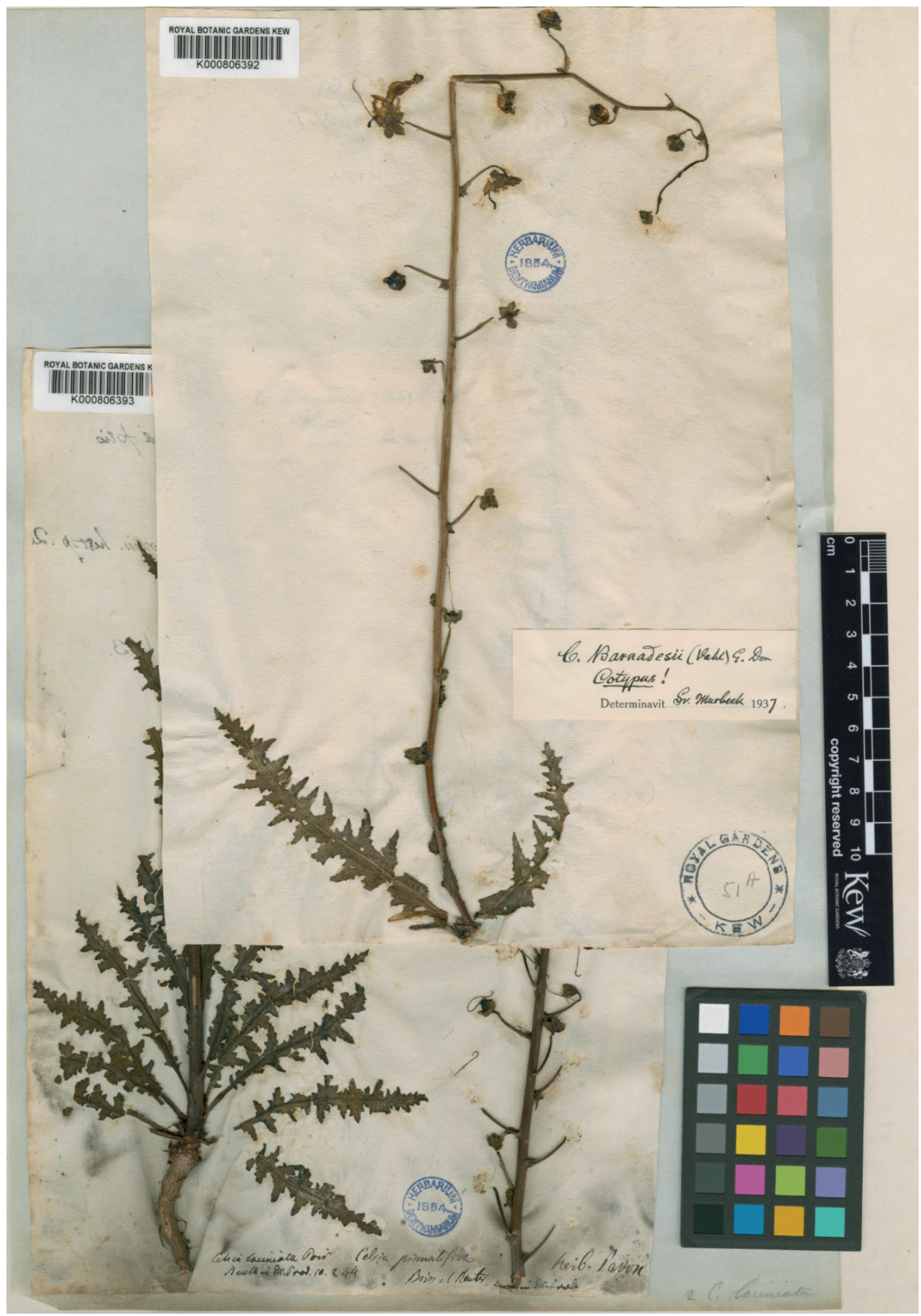

Figura 1. Isolectótipo de Verbascum barnadesii Vahl (K000806392). Herbario K, reproducido con permiso. 
bien conservados, que parecen pertenecer a la misma planta; por un lado una parte basal, un tallo con hojas basales y medias, y un segundo fragmento que corresponde a la inflorescencia (Fig. 2). En la base de estos dos fragmentos aparece anotado, en diferentes grafías, lo siguiente: "Celsia laciniata Pour. / [ilegible?] in DC. Prodr. 10. 244 / Celsia pinnatifida / Boiss. et Reuter / herb. Pavon" (imagen disponible en: https://plants.jstor.org/stable/viewer/10.5555/ al.ap.specimen.k000806393). Además, en el reverso de esta hoja (K000806393) aparece anotado lo siguiente: "Blattaria orientalis agrimonia folio / F. cor. 8 /vel potius / Blattaria ragusina incana multifida Moris hist. p. 2 / pag. 490 , n. 9 sent. 5 t. 10 f. 9 / Bocc var. plant. sic. p 61. t. 31. f. 3 / Tourn. 148 ", y en la parte inferior de esta cara de la hoja se puede leer lo siguiente: "Maj.-Iun. 1757 / Ex Colliculis de Hincapie ad dexteram viae rectoris / ab opp. Ortaleza ad Praedium Vust. la Moraleja / Tract. Matritens." (imagen disponible en: https://plants. jstor.org/stable/viewer/10.5555/al.ap.specimen. k000806393?page $=2$ ). Este interesante material tal vez llegó a Londres a través del envío de pliegos de Barnades a Joseph Banks (N. Ibáñez, com. pers.).

Por otra parte, en el herbario $\mathrm{C}$ se conserva un espécimen de esta especie (C10018908), compuesto por cuatro hojas y una inflorescencia con flores algo deterioradas. El fragmento está acompañado de una etiqueta de revisión de Murbeck, en la que se lee: "Specimen originarium! / C. Barnadesii (Vahl) G. Don / t. Sv. Murb. 1921" (imagen disponible en: https://plants.jstor.org/stable/ viewer/10.5555/al.ap.specimen.c10018908; http:// www.daim.snm.ku.dk/digitized-type-collectiondetails-simple?catno=LNR-6174). Además, en el reverso del pliego aparece anotado lo siguiente: "Verbascum Barnadesii / Dr. Barnades" y "Maj.Iun. 1757 / Ex Colliculis de Hincapie ad dexteram viae rectoris / ab opp. Ortaleza ad Praedium st. la Moraleja / Tract. Matritens." (imagen disponible en: https://plants.jstor.org/stable/viewer/10.5555/ al.ap. specimen $\cdot$ c10018908?page $=2$ ). Esta anotación coincide exactamente con lo que aparece en el pliego de Kew y con lo publicado en el protólogo "Habitat in colliculis de Hincapie versus oppidum Ortaleza tractus Madritensis". Asimismo, el material corresponde sin duda al espécimen que fue enviado por Miquel Bernades i Mainader a Martin Vahl, como se comunicaba en el protólogo "Specimen mecum communicavit Cel. Dn. Barnades filius inventoris" y fuera advertido por Murbeck (1921: 4-6). En esta publicación de Murbeck, se reprodujo además una imagen de este espécimen, pero lamentablemente no se concretó ninguna tipificación del nombre, tal vez por considerar Murbeck que el especimen $C$ fue el único materal que utilizó Vahl y quizá por desconocer a la vez la existencia del material conservado en $\mathrm{K}$, ya que éste fue revisado por él mismo años más tarde, en 1937.

Por último, mencionar que Ibáñez (2006) e Ibáñez et al. (2009) recogen un pliego de Barnades de una especie de Verbascum, con el número 658 (BC-Barnades 658), con la siguiente anotación: "Verbascum (manuscrito de Miquel Barnades i Mainader) sinuatum (manuscrito de Miquel Barnades i Claris) L. / Septembr. 1747 / Ex incultis pagi Ambroz sub Templo. dit. Madrit." (m. de Miquel Barnades i Claris). Este pliego está en la actualidad identificado con el código de barras BC-972766, y contiene dos hojas basales y un fragmento de tallo con hojas pero sin flores. El pliego tiene la siguiente anotación "Verbascum Linn." en la parte superior del especimen, y en el reverso del pliego aparece anotado lo siguiente: "Verbascum foliis radicalibus oblongis sinuates undatis tomentosis, caulinis cordatis / amplexicaulibus nudiusculis Linn. spec. plant. p. 178 n. ${ }^{\circ}$ 6. // Verbascum foliis pinnato-sinuatis hirsutis, subtus tomentosis Roy. Flor. Leid. p. 421. / Sauvages Meth. Folior. pag. 276. num. ${ }^{\circ}$ 52. // Verbascum nigrum foliis papaveris corniculati C. Bauh. pin. 240. Tourn. 147. // Camerar. hist. pag. et Tab. 403. // sinuatum" (en la parte superior de la hoja), y "Septembr. 1747. / Ex incultis pagi Ambroz sub Templo. dit. Matrit." (en la parte inferior de la hoja) (Figs. 3 y 4). Así, este material no corresponde a un duplicado de $V$. barnadesii de los especímenes conservados en $\mathrm{K}$ y C. Además, el especimen que contiene puede ser identificado como perteneciente a $V$. sinuatum $\mathrm{L}$.

Finalmente, indicar que, al parecer no se conserva ningún material original de $V$. barnadesii en el herbario personal de Barnades (N. Ibáñez, com. pers.).

En conclusión, según la información que hemos manejado, el tipo de este nombre no ha sido formalmente designado hasta el momento (véase Murbeck, 1921; Benedí \& Montserrat, 1985; Benedí, 2009). En consecuencia, designamos como lectótipo del nombre Verbascum barnadesii el espécimen conservado en $\mathrm{C}$ (con código de barras C10018908), el cual fue empleado por Vahl para 


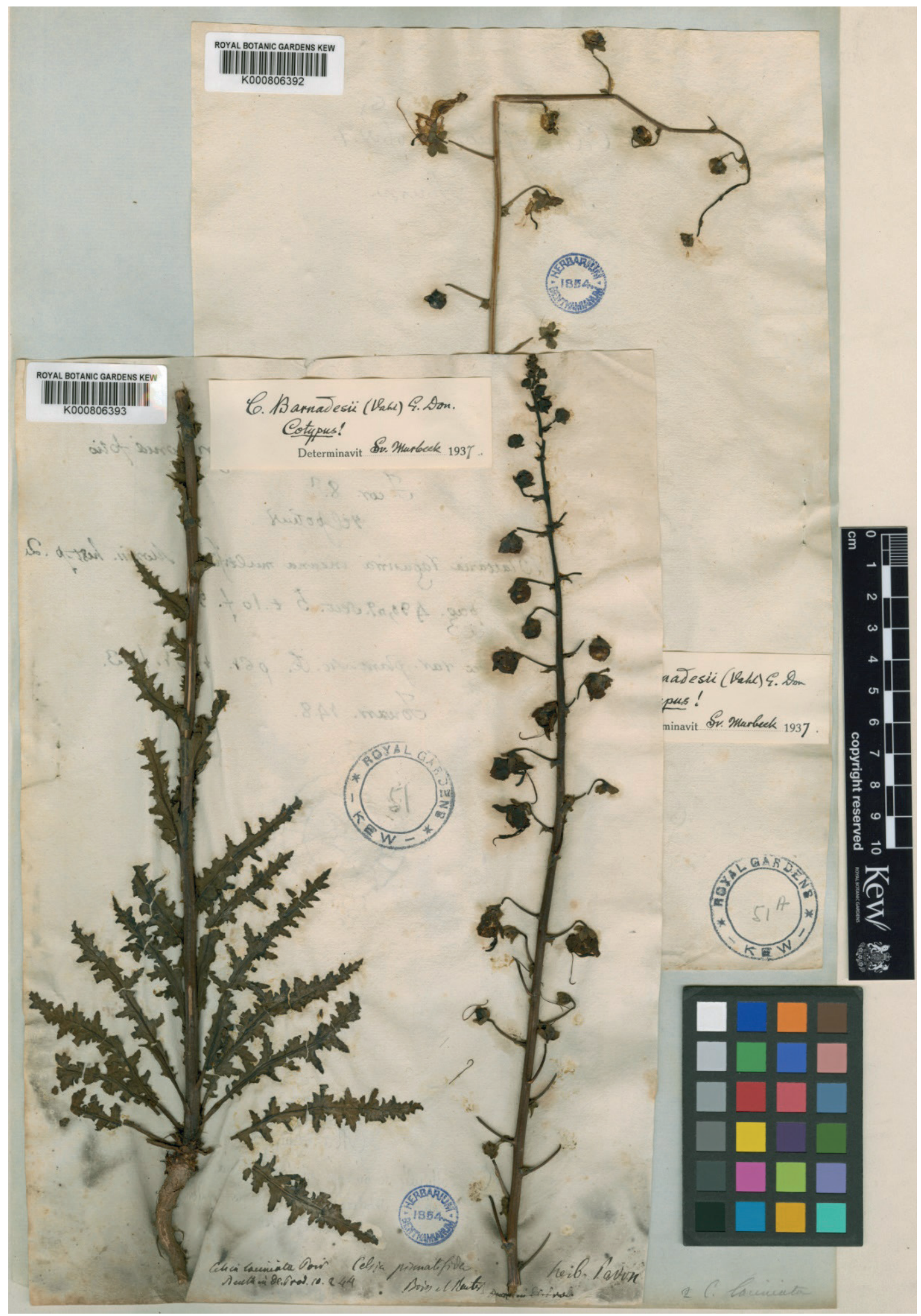

Figura 2. Isolectótipo de Verbascum barnadesii Vahl (K000806393). Herbario K, reproducido con permiso. 
la descripción de su especie. En consecuencia, los especímenes (o especimen) codificado/s como K000806393 y K000806392 deben ser tratados como duplicados y por tanto como isolectótipos, ya que es material que contiene la misma anotación de Barnades "Maj.-Iun. 1757 / Ex Colliculis de Hincapie ad dexteram viae rectoris / ab opp. Ortaleza ad Praedium Vust. la Moraleja / Tract. Matritens.”.

Verbascum barnadesii Vahl, Symb. Bot. 2: 39. 1791 $\equiv$ Celsia barnadesii (Vahl) G. Don, Gen. Hist. 4: 499. 1837-1838

Ind. loc.: "Habitat in colliculis de Hincapie versus oppidum Ortaleza tractus Madritensis. Specimen mecum communicavit Cel. Dn. Barnades filius inventoris".

Lectotypus (hic designatus): Ex Colliculis de Hincapie ad dexteram viae brevioris ab opp. Ortaleza ad Praedium Vust. la Moraleja Tract. Matritens. V/ VI-1757, Miquel Barnades i Mainader, C (código de barras C10018908). Isolectotypi: K000806392 (Fig. 1) y K000806393 (Fig. 2).

\section{Verbascum fontqueri}

Benedí \& Montserrat (1985: 104) mencionan como "lectótipo" de $V$. fontqueri un material conservado en BC (en la actualidad con el código de barras BC 43678) (Fig. 5), y en concreto indican lo siguiente: "al plec [BC 43678 hi ha dos exemplars, el lectotypus és el de la dreta, l'altre és un isotypus". Este tipo fue considerado posteriormente por Benedí (2009). El pliego BC 43678, además de contener dos plantas completas, conserva una etiqueta manuscrita original de Font Quer, en la que se puede leer: "Celsia valentina F.Q. / -typus- / in Regno Valentino, c. "La Barraca de Valldigna"/ inter ceratonias, $350 \mathrm{~m}$ alt., 1. "Barranco la Falzia" / leg. F. Q., 5 junii 1923" (Fig. 5). Una estupenda iconografía de la planta seleccionada como "lectótipo" fue realizada por Eugeni Serra i Ràfols (1919-1999) y aparece publicada en el trabajo de Benedí \& Montserrat (1985: 105).

No obstante, según lo indicado en el Art. 9.12 del ICN (Turland et al., 2018), desafortunadamente este "lectótipo" no sería efectivo, ya que Font Quer (1926: 56) cita en el protólogo de Celsia valentina [sinónimo sustituido del nombre nuevo propuesto por Benedí \& Montserrat (1985: 103) como V. fontqueri], dos recolecciones concretas: "Hab. in agris, praecipue inter ceratonias, virgultisque, etc, Regni
Valentini, pr. La Barraca de Valldigna, $l$. "Barranc de la Falzia" dicto, ubi J. 26 majii 1923, inveni; Gros non procul a La Barraca, loco "Serra de les Agulles", $d$. 7 junii, legit.", es decir, una realizada el 26 de mayo de 1923, con material recolectado en "pr. La Barraca de Valldigna, 1. Barranc de la Falzia", y otra el 7 de junio [de 1923] procedente de "Serra de les Agulles"; aunque el año de esta segunda recolección no aparece en el protólogo, sí que está presente en una etiqueta de un pliego de herbario (BC 43676).

En consecuencia, los materiales que componen estas dos recolecciones deben ser considerados como síntipos (Art. 9.6 del $I C N$ ), y por tanto tienen prioridad en la designación del lectótipo frente al material original no citado en el protólogo (Art. 9.12), como es el caso del especimen conservado en el pliego BC 43678 considerado por Benedí y Montserrat, ya que pertenece a una recolección no citada en el protólogo, con fecha de 5 de junio de 1923.

Respecto a los síntipos, se conserva en varios herbarios material procedente de "pr. La Barraca de Valldigna, Barranc de la Falzia" y recolectado el 26 de mayo de 1926, como por ejemplo BC 82585, BC 82590, BC 82591, BC 43677 (dos hojas), BCN 60388 (ex BCF 31669), BCN 63173 (ex BCF), BCN 7754 (ex BCC), GH00078140, K000806391, S10-27232. Estos pliegos contienen plantas muy completas y en buen estado de conservación, acompañadas en todos los casos por la misma etiqueta impresa, en la que se puede leer: "Institutus Botanicus Barcinonensis / Flora Iberica Selecta / Cent. I / Dec. 1934 / 79. Celsia valentina Font Quer / in Butll. Inst. Cat. Hist. Nat., 1926, p. 56. / Valentia: in agris, praecipue inter ceratonias, virgultisque $/ 1$. Barranc / de la Falzia, prope La Barraca de Valldigna. Cotypus. / Leg. Font Quer, 26 maji 1923”.

En relación al otro material síntipo, el procedente de "Serra de les Agulles", recolectado el 7 de junio [de 1923], se conserva un pliego en BC (BC 43676), que contiene un tallo con hojas, frutos y una flor, y una etiqueta manuscrita por Font Quer en la que se puede leer lo siguiente: "Celsia valentina F. Q. / in Regno Valentino, Serra de les Agulles, c.Valldigna / Gros, 7 Junii 1923 / F. Q." (Fig. 6). Este pliego fue citado en el apartado "Localitats estudiades" por Benedí \& Montserrat (1985: 105).

Entre todo el material arriba comentado, se propone como lectótipo del nombre Verbascum fontqueri el espécimen BC 82585 (Fig. 7), siendo por 


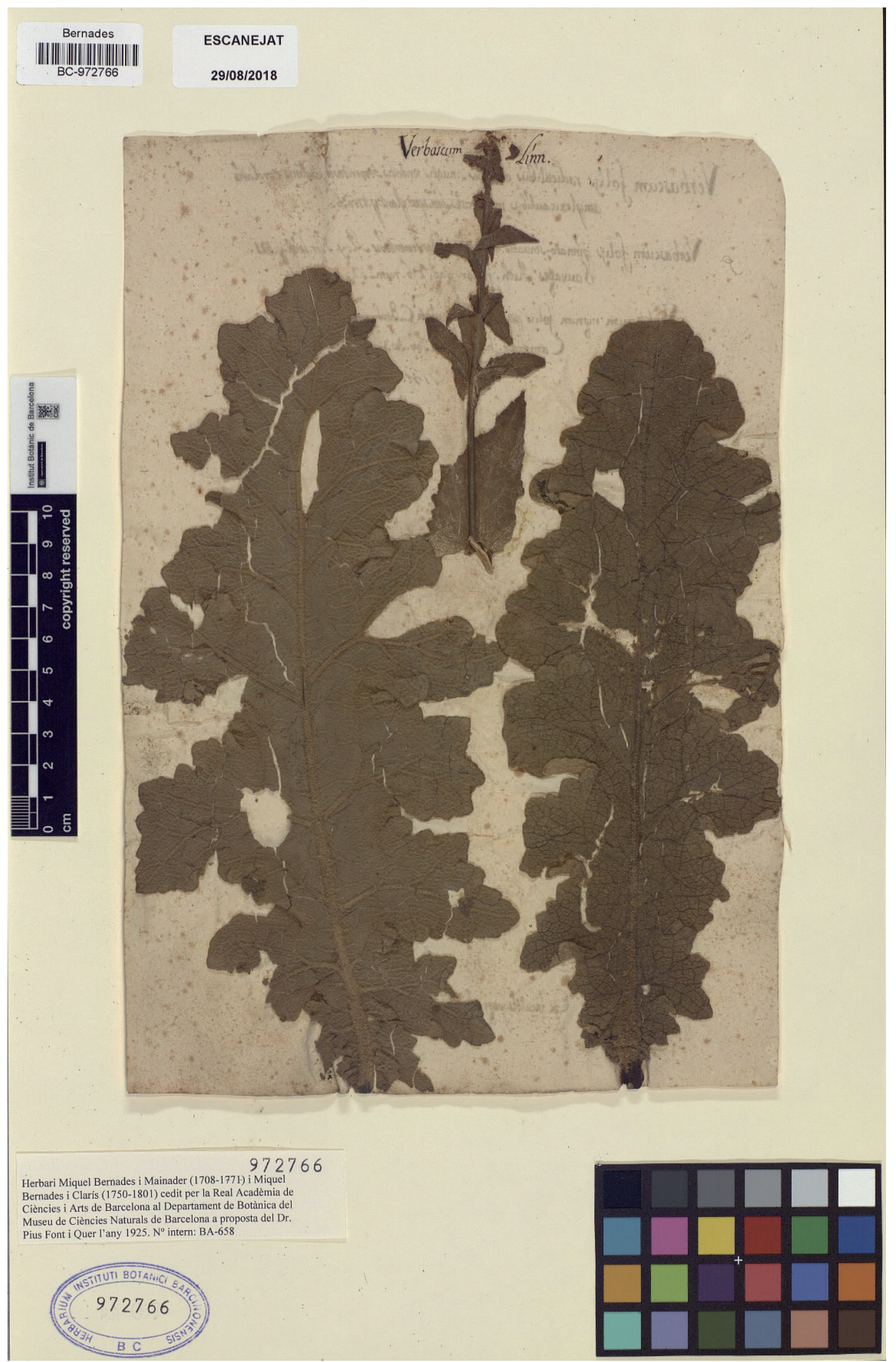

Figura 3. Anverso del pliego de Miquel Barnades (BC-Barnades 658; código de barras BC-972766) con material de Verbascum sinuatum. Herbario BC, reproducido con permiso. 


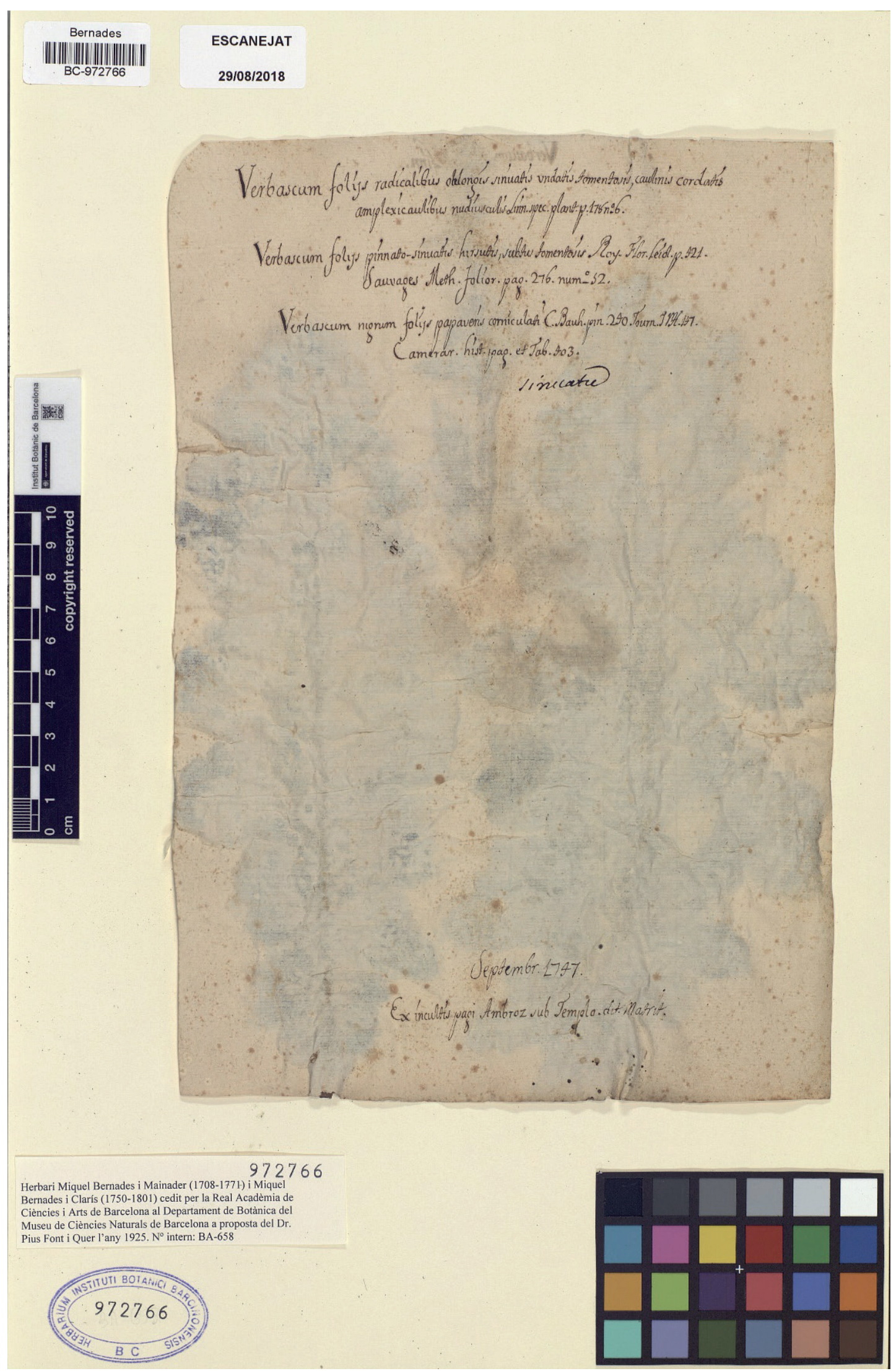

Figura 4. Reverso del pliego de Miquel Barnades (BC-Barnades 658; código de barras BC-972766) con material de Verbascum sinuatum. Herbario BC, reproducido con permiso. 


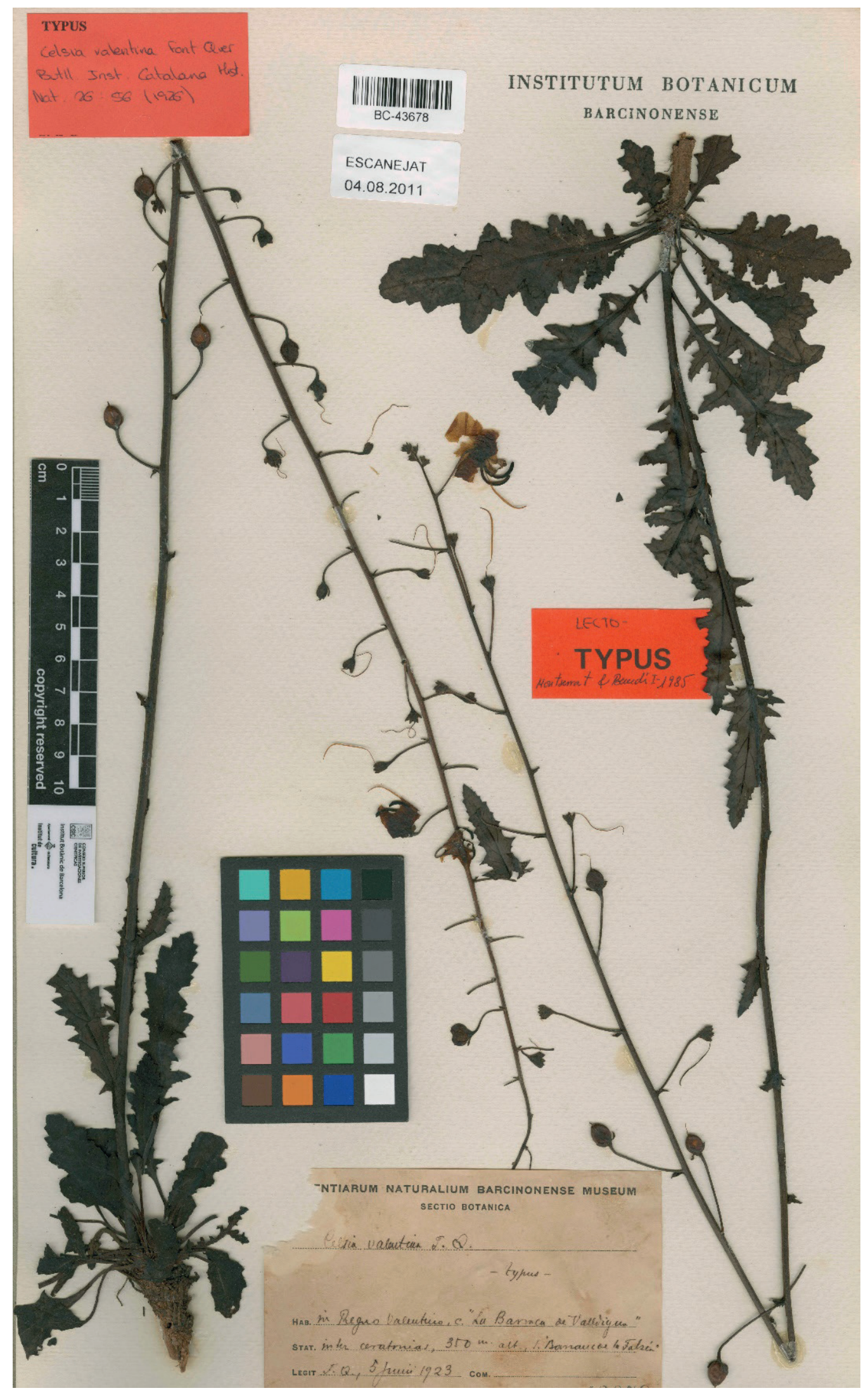

Figura 5. Especimen propuesto por Benedí \& Montserrat (1985: 104) como "lectótipo" de Verbascum fontqueri Benedí \& J. M. Monts. (BC 43678). Herbario BC, reproducido con permiso. 


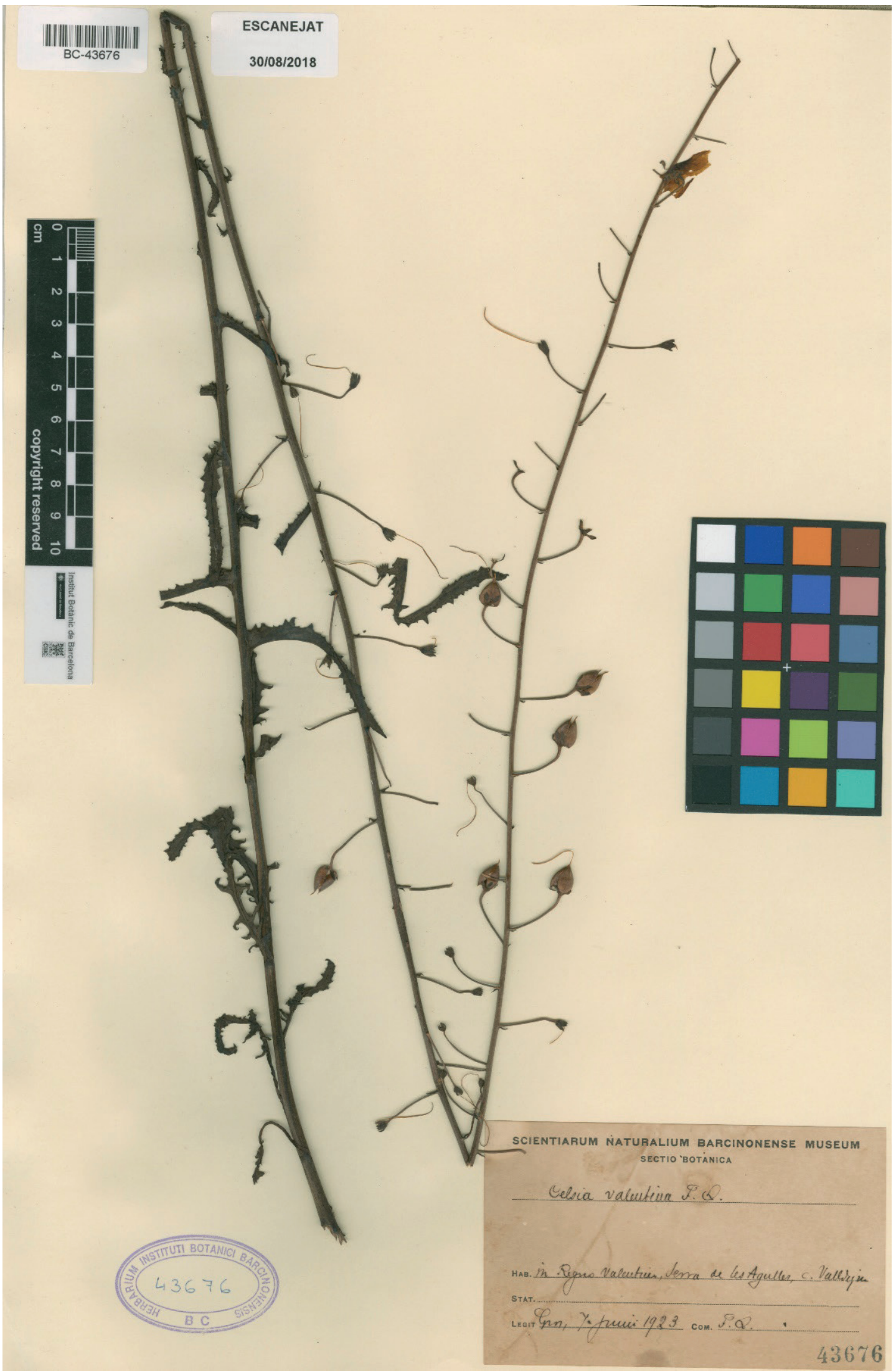

Figura 6. Material original y síntipo de Verbascum fontqueri Benedí \& J. M. Monts., procedente de "Serra de les Agulles", recolectado el 7 de junio de 1923 (BC 43676). Herbario BC, reproducido con permiso. 


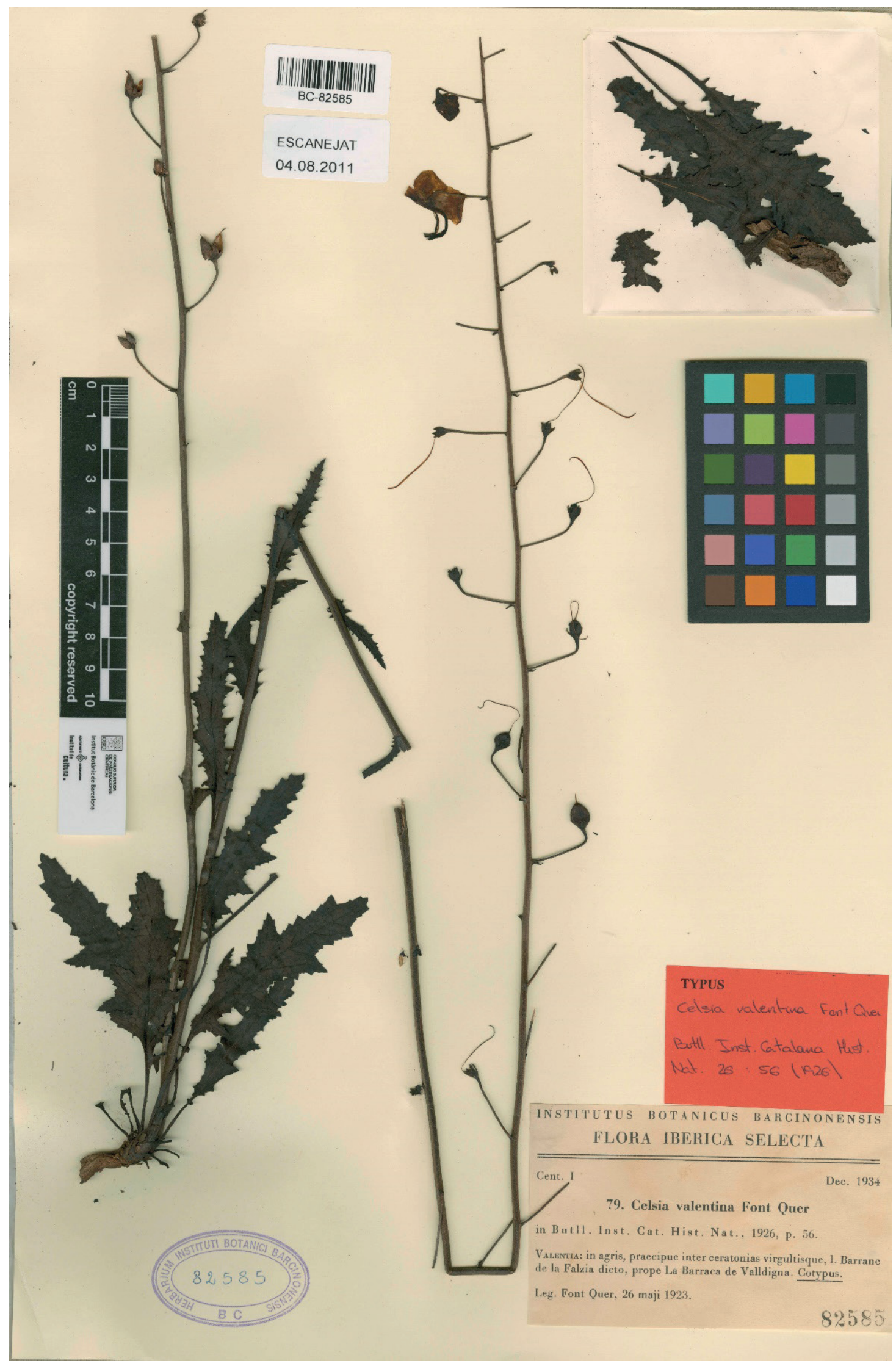

Figura 7. Lectótipo de Verbascum fontqueri Benedí \& J. M. Monts. (BC 82585). Herbario BC, reproducido con permiso. 
tanto isolectótipos los especímenes conservados en los siguientes pliegos: BC 82590, BC 82591, BC 43677 (dos hojas), BCN 60388 (ex BCF 31669), BCN 63173 (ex BCF), BCN 7754 (ex BCC), GH00078140, K000806391, S 10-27232. Aunque esta designación no cambia el uso ni el concepto actual del nombre $V$. fontqueri respecto a la propuesta de Benedí y Montserrat, ha sido necesario realizarla para legitimar su tipo nomenclatural de acuerdo al $I C N$.

Verbascum fontqueri Benedí \& J. M. Monts., Collect. Bot. (Barcelona) 16: 103. 1985 [nom. nov.] $\equiv$ Celsia valentina Font Quer in Butll. Inst. Catalana Hist. Nat. 26: 56.1926 [syn. subst.], non Verbascum valentinum Burnat \& Barbey, Notes Voy. Bot. Baléares et Valence: 50. 1882

$\equiv$ Verbascum barnadesii Vahl. subsp. valentinum (Font Quer) O. Bolòs \& Vigo, Collect. Bot. (Barcelona) 14: 96. 1983.

Ind. loc.: "Hab. in agris, praecipue inter ceratonias, virgultisque, etc., Regni Valentini, pr. La Barraca de Valldigna, 1. "Barranc de la Falzia" dicto, ubi d. 26 majii 1923; Gros non procul a La Barraca, loco "Serra de les Agulles", d. 7 junii, legit".

Lectotypus (hic designatus): Valentia: in agris, praecipue inter ceratonias, virgultisque 1 . Barranc de la Falzia, prope La Barraca de Valldigna, 26V-1923, P. Font i Quer, BC 82585 (Fig. 7). Isolectotypi: BC 82590, BC 82591, BC 43677 (dos hojas: BC-43677 + BC-43677-a), BCN 60388 (ex BCF 31669), BCN 63173 (ex BCF), BCN 7754 (ex BCC), GH00078140 (https://plants.jstor.org/stable/viewer/10.5555/al.ap.specimen.gh00078140), K000806391 (http://apps.kew.org/herbcat/getImage.do?imageBarcode=K000806391; https://plants. jstor.org/stable/viewer/10.5555/al.ap.specimen. k000806391), S 10-27232 (https://plants.jstor. org/stable/viewer/10.5555/al.ap.specimen.s1027232 ?loggedin=true).

\section{AGRADECIMIENTOS}

A N. Ibáñez, E. Farràs y N. Nualart (Herbario BC, Institut Botànic de Barcelona), R. Guàrdia (Centre de Documentació de Biodiversitat Vegetal, Universitat de Barcelona) y a O. Ryding y N. Rønsted (Herbarium C, University of Copenhagen) por la ayuda en el estudio de los pliegos de herbario conservados en $\mathrm{BC}$ y $\mathrm{C}$, respectivamente.

\section{REFERENCIAS BIBLIOGRÁFICAS}

Aguilella, A., Fos, S. \& Laguna, E. (Eds.) 2010. Catálogo valenciano de especies de flora amenazadas (Colección Biodiversidad, 18). Conselleria de Medi Ambient, Aigua, Urbanisme i Habitatge, Generalitat Valenciana, Valencia.

Benedí, C. 2009. Verbascum L. In: Benedí, C., Rico, E., Güemes, J. \& Herrero, A. (Eds.), Flora iberica 13. Real Jardín Botánico, CSIC, Madrid: 49-71.

Benedí, C. \& Montserrat, J. M. 1985. Notes taxonòmiques i nomenclatures sobre el gènere Verbascum L. (Celsia L.) a la Península Ibèrica i a les Illes Balears. Collectanea Botanica 16: 101-112.

Benedí, C., Orell, J. \& Orell Jaquotot, J. J. 1989. Notes taxonòmiques, nomenclaturals i corològiques de les espècies de Verbascum L. (Scrophulariaceae) a Mallorca. Butlletí de la Institució Catalana d'Història Natural 57: 61-64.

Ferguson, I. K. 1972. Verbascum L. In: Tutin, T. G., Heywood, V. H., Burges, N. A., Moore, D. M., Valentine, D. H., Walters, S. M. \& Webb, D. A. (Eds.), Flora europaea 3. Cambridge University Press, Cambrigde: 205-216.

Font Quer, P. 1926. De flora occidentale adnotationes, II. Butlletí de la Institució Catalana d'Història Natural 26: 53-57.

Generalitat Valenciana 2013. Orden 6/2013, de 25 de marzo, de la Consellería de Infraestructuras, Territorio y Medio Ambiente, por la que se modifican los listados valencianos de especies protegidas de flora y fauna. Diari Oficial de la Generalitat Valenciana 6996: 8682-8690.

Heywood, V. H. 1993. Flowering plants of the world. Oxford University Press, New York.

Ibáñez, N. 2006. Estudis sobre cinc herbaris històrics de l'Institut Botànic de Barcelona. Tesi Doctoral, Universitat de Barcelona.

Ibáñez, N., Soriano, I. \& Montserrat, J. M. 2009. L’herbari Bernades a l'Institut Botànic de Barcelona (BC). Collectanea Botanica 28: 31-63. https://doi.org/10.3989/collectbot.2008.v28.002

Judd, W. S., Campbell, C. S., Kellogg, E. A., Stevens, P. F. \& Donoghue, M. J. 2007. Plant systematics: a phylogenetic approach. Sinauer, Sunderland.

Murbeck, S. 1921. Sur quelques espèces nouvelles ou critiques du genre Celsia, etc. Lunds Universitets Arsskrift, n. f. avd. 2 17: 1-18.

Murbeck, S. 1925. Monographie der Gattung Celsia. Lunds Universitets Arsskrift, n. f. avd. 2 22: 1-241.

Murbeck, S. 1933. Monographie der Gattung Verbascum. Lunds Universitets Arsskrift, n. f. avd. 2 29: 1-630.

Murbeck, S. 1939. Weitere Studien über die Gattungen Verbascum und Celsia. Lunds Universitets Arsskrift, n. f. avd. 2 35: 1-71.

Swingle, W. T. 1912. Clastotypes, clonotypes, and spermotypes, means for multiplying botanical name specimens. Journal of the Washington Academy of Sciences 2: 344.

Thomas, O. 1893. Suggestions for the more definite use of the word "Type" and its compounds, as denoting specimens of a greater or less degree of authenticity. Proceedings of the Zoological Society of London 1893: 241-242. https://doi. org/10.1248/yakushi1881.1893.132 241

Turland, N. J., Wiersema, J. H., Barrie, F. R et al. (Eds.) 2018. International Code of Nomenclature for algae, fungi, and plants (Shenzhen Code) (Regnum Vegetabile, 159). Koeltz Botanical Books, Glashütten. https://doi.org/10.12705/Code.2018

Vahl, M. 1791. Symbolae botanicae 2. N. Möller \& filius, Copenhagen. 